\title{
Blue-Detuned Magneto-Optical Trap
}

\author{
K. N. Jarvis, ${ }^{*}$ J. A. Devlin, T. E. Wall, B. E. Sauer, and M. R. Tarbutt \\ Centre for Cold Matter, Blackett Laboratory, Imperial College London, Prince Consort Road, London SW7 2AZ, United Kingdom
}

(Received 11 October 2017; published 20 February 2018)

\begin{abstract}
We present the properties and advantages of a new magneto-optical trap (MOT) where blue-detuned light drives "type-II" transitions that have dark ground states. Using ${ }^{87} \mathrm{Rb}$, we reach a radiation-pressurelimited density exceeding $10^{11} \mathrm{~cm}^{-3}$ and a temperature below $30 \mu \mathrm{K}$. The phase-space density is higher than in normal atomic MOTs and a million times higher than comparable red-detuned type-II MOTs, making the blue-detuned MOT particularly attractive for molecular MOTs, which rely on type-II transitions. The loss of atoms from the trap is dominated by ultracold collisions between $\mathrm{Rb}$ atoms. For typical trapping conditions, we measure a loss rate of $1.8(4) \times 10^{-10} \mathrm{~cm}^{3} \mathrm{~s}^{-1}$.
\end{abstract}

DOI: 10.1103/PhysRevLett.120.083201

The magneto-optical trap (MOT) [1] is an essential tool for a wealth of scientific and technological applications of ultracold atoms, including tests of fundamental physics, studies of ultracold collisions and quantum degenerate gases, advances in frequency metrology, and the development of commercial cold atom instruments, such as gravimeters and clocks. Similarly, applications of ultracold molecules [2] are sure to be advanced by recent demonstrations of molecular MOTs [3-6]. All previous MOTs have been made using light red detuned from the atomic or molecular transition. Red detuning is required for Doppler cooling, the primary cooling mechanism in a MOT. SubDoppler cooling also requires red-detuned light when the excited-state angular momentum $\left(F^{\prime}\right)$ exceeds that of the ground state $(F)$. These transitions are called type I and are used in almost all MOTs. Atomic MOTs have also been made using type-II transitions that have $F^{\prime} \leq F$ [1,7-9]. These MOTs tend to produce relatively hot clouds with low density, so have not been much used. In molecular MOTs, however, type-II transitions must be used to avoid rotational branching [10]. Like their atomic counterparts, these MOTs also exhibit low density and high temperature, and this has stimulated renewed interest in the cooling and trapping mechanisms at work [11-13] and in methods to increase the phase-space density obtained in type-II MOTs.

Here, we increase the phase-space density through a conceptually simple, but counterintuitive, change to the normal procedure-we use light that is blue detuned from the atomic transition. We demonstrate a blue-detuned type-II MOT of ${ }^{87} \mathrm{Rb}$ with a density exceeding $10^{11} \mathrm{~cm}^{-3}$

Published by the American Physical Society under the terms of the Creative Commons Attribution 4.0 International license. Further distribution of this work must maintain attribution to the author(s) and the published article's title, journal citation, and DOI. and a temperature below $30 \mu \mathrm{K}$. The dimensionless phasespace density is $6 \times 10^{-6}$, about a million times higher than for a red-detuned type-II MOT [9]. Indeed, this phasespace density is considerably higher than usually achieved in a normal type-I MOT and is similar to that achieved in the best dark spontaneous-force optical trap (SPOT) MOTs [14,15].

Figure 1 illustrates the principle of the blue-detuned MOT. The $z$ axis is defined by the magnetic field $\vec{B}$. Lasers drive the $F \rightarrow F^{\prime}=1 \rightarrow 1$ and $2 \rightarrow 2 D 2$ transitions in ${ }^{87} \mathrm{Rb}$. Both are type-II transitions. Provided the detunings are much smaller than the hyperfine intervals, so that each frequency component primarily drives only one transition, the position-dependent force in a MOT is unchanged when the detuning and the handedness of the light are both reversed, as illustrated in Fig. 1(b). For our level scheme, trapping is obtained when the light is red detuned and the restoring beam, propagating toward $-z$, is polarized to drive $\Delta m_{F}=-1$ transitions, or when blue detuned and polarized to drive $\Delta m_{F}=+1$. By contrast, the velocitydependent force, shown in Fig. 1(c), changes sign when the detuning is reversed, irrespective of polarization choice. For type-I transitions, the Doppler and polarizationgradient components of the force have the same sign, both providing cooling for red-detuned light. For type-II transitions, these two components of the force have opposite signs, so sub-Doppler cooling requires blue-detuned light. Sub-Doppler cooling on type-II transitions has been applied to atoms [16-19] and molecules [5] in optical molasses. This cooling relies on coupling between dark and bright states, induced either by motion through the changing polarization of the light field [20] or by an applied magnetic field [21-23]. A recent theoretical study [13] of these polarization gradient forces in a 3D type-II molasses shows that they are strong and can act over a wide velocity range. This can be seen in Fig. 1(c), where sub-Doppler 

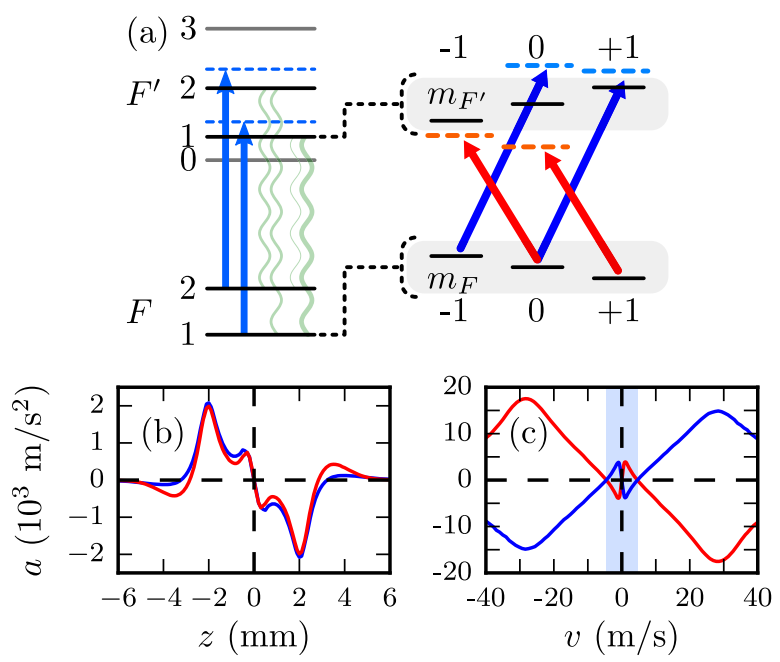

FIG. 1. (a) Relevant energy levels and the transitions we drive. Relative decay rates are indicated by the thickness of the green wavy lines. The Zeeman structure of the $1 \rightarrow 1$ transition is shown. Trapping is obtained when the light is red detuned and polarized to drive $\Delta m_{F}=-1$ transitions or blue detuned and polarized to drive $\Delta m_{F}=+1$. (b) Position-dependent acceleration curves for these two cases, showing that, near the trap center, the force is unchanged when the polarization and detuning are reversed. For large Zeeman splittings, the symmetry is broken by the other hyperfine components. (c) Velocity-dependent acceleration curves for the two cases. For positive detuning (blue curve) there is polarization-gradient cooling but Doppler heating, while for negative detuning (red curve), the opposite is true. (b) and (c) are calculated for a six-beam MOT using optical Bloch equations [13] that take into account the ${ }^{87} \mathrm{Rb}$ level structure and Zeeman shifts, as well as the two laser frequency components of light. The parameters used were $\delta f_{11}= \pm 11.5 \mathrm{MHz}, \delta f_{22}= \pm 26 \mathrm{MHz}, I_{t}=113 \mathrm{~mW} / \mathrm{cm}^{2}$, and $B^{\prime}=87 \mathrm{G} / \mathrm{cm}$.

cooling is effective for speeds below a critical velocity, $v_{\mathrm{c}} \approx 4.5 \mathrm{~m} / \mathrm{s}$, where the force crosses zero. This is the mean speed for a thermal distribution at $80 \mathrm{mK}$. The study also showed that, while polarization-gradient forces in type-I systems are suppressed by magnetic fields, for type-II systems, they remain strong over the whole range of fields atoms explore in a typical MOT. Based on these findings, it was suggested [13] that a blue-detuned type-II MOT cooled exclusively by polarization-gradient forces should be feasible and would confine atoms just as strongly as its red-detuned counterpart, while cooling to far lower temperature. Here, we demonstrate that idea.

We form two sets of MOT beams, $A$ and $B$, with opposite handedness, and can switch between them using acoustooptic modulators as optical switches. All beams have Gaussian intensity distributions, and the $1 / e^{2}$ radius is $9.7 \mathrm{~mm}$ for set $A$ and $5.8 \mathrm{~mm}$ for set $B$. Using beams $A$, we load a standard red-detuned type-I ${ }^{87} \mathrm{Rb}$ MOT from the background vapor produced by a dispenser. Once the desired number of atoms has been loaded, up to a maximum of $10^{9}$, the light is switched off, the polarization handedness is reversed by switching to beams $B$, the laser frequencies are stepped via an offset lock, so that one frequency component is detuned by $\delta f_{11}$ from the $1 \rightarrow 1$ transition and the other by $\delta f_{22}$ from $2 \rightarrow 2$, and then, after a settling period of $2 \mathrm{~ms}$, the light is turned back on, producing a total peak intensity at the MOT of $I_{\mathrm{t}}$, divided equally between the two frequency components. The axial magnetic field gradient $B^{\prime}$ is switched to a new value during this period.

Atoms are detected by absorption imaging using light tuned close to the $2 \rightarrow 3$ transition. The fraction recaptured in the blue-detuned MOT is close to $100 \%$ over a wide range of parameters. Nevertheless, the MOT fluorescence decreases to $20-30 \%$ of the type-I MOT fluorescence. This reduction in scattering rate is expected because the subDoppler cooling mechanism relies on atoms spending part of their time in dark states [20]. We only observe trapping when $\delta f_{11}>0$, as expected for the reversed polarization handedness. We observe a MOT for both positive and negative $\delta f_{22}$, but the temperature is higher and the density lower when $\delta f_{22}<0$. This shows that the $1 \rightarrow 1$ transition is mainly responsible for the MOT, which makes sense since $F^{\prime}=1$ decays to $F=1$ with probability $5 / 6$, whereas $F^{\prime}=2$ decays to the two ground states with equal probability. We have measured the state distribution and find that, for typical parameters, $85 \%-90 \%$ of ground-state atoms are in $F=1$.

Figure 2 shows how the peak number density $n_{0}$ varies with the number of trapped atoms $N$ for various $B^{\prime}$. The density initially increases linearly with $N$ and can be described by $n_{0}=N / V_{0}$, where $V_{0}$ is the volume for small $N$. For higher $N$, the density saturates toward a maximum value, which we attribute to photon rescattering - a photon scattered by one atom can be rescattered by another, introducing an effective repulsive force that counteracts the MOT confinement, giving a maximum attainable density $n_{\max }$ independent of $N[24,25]$. We fit the data to the model $n_{0}=N /\left(V_{0}+N / n_{\max }\right)$, with $V_{0}$ and $n_{\max }$ as free parameters. This model fits well over the range of $N$ and $B^{\prime}$ explored. We find a linear relationship between $n_{\max }$ and $B^{\prime}$, as expected [26], with a gradient of

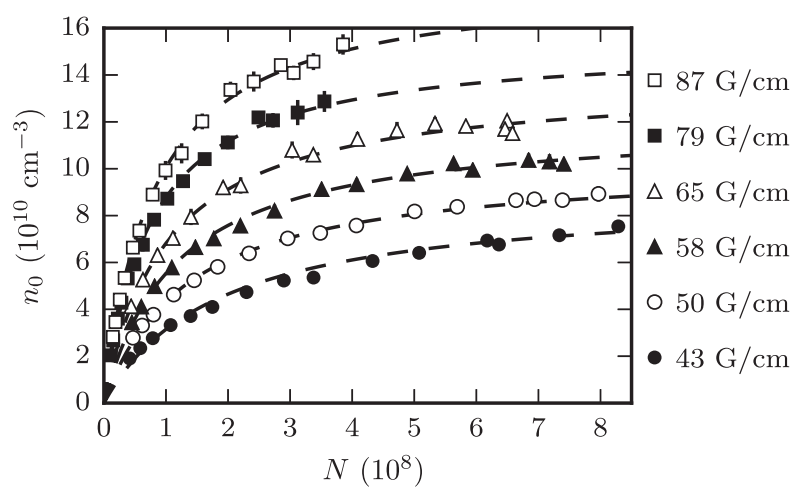

FIG. 2. Peak number density $\left(n_{0}\right)$ versus the number of atoms in the MOT $(N)$ for a range of magnetic field gradients. Dashed lines are fits to $n_{0}=N /\left(V_{0}+N / n_{\max }\right)$. 

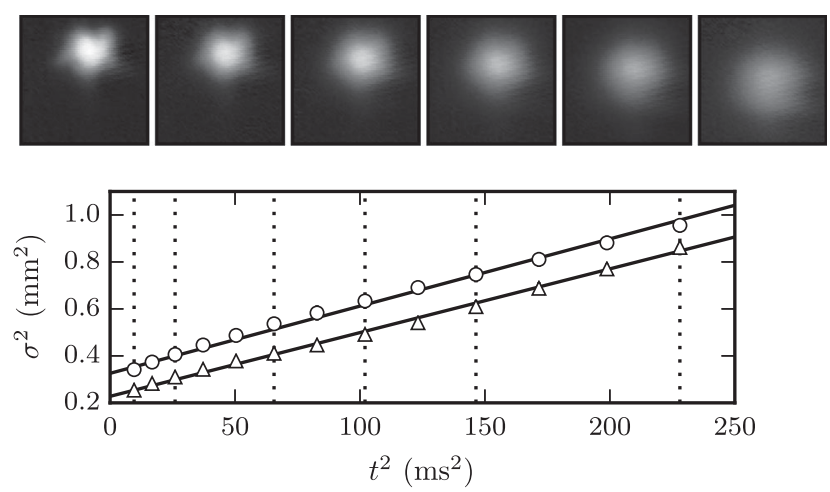

FIG. 3. Ballistic expansion of atoms released from the blue-detuned MOT. The parameters are $\delta f_{11}=35 \mathrm{MHz}$, $\delta f_{22}=11.5 \mathrm{MHz}$, and $B^{\prime}=87 \mathrm{G} / \mathrm{cm}$. The MOT is loaded at $I_{t}=130 \mathrm{~mW} / \mathrm{cm}^{2}$ and $I_{t}$ is then reduced to $22 \mathrm{~mW} / \mathrm{cm}^{2}$ for $5 \mathrm{~ms}$ before the atoms are released. Images are $5 \times 5 \mathrm{~mm}$ and correspond to the times indicated by the dotted lines in the lower panel. The lower panel shows the rms widths in the radial (circles) and axial (triangles) directions. The fitted temperatures are $T_{\rho}=30(1)$ and $T_{z}=29(1) \mu \mathrm{K}$.

$2.1(1) \times 10^{9} \mathrm{~cm}^{-3} /(\mathrm{G} / \mathrm{cm})$. Provided the MOT temperature is independent of $B^{\prime}$, which it is over this range (see below), and the restoring force is linear in the displacement with a slope proportional to $B^{\prime}$, we expect the relationship $V_{0} \propto\left(B^{\prime}\right)^{-3 / 2}$. We measure $V_{0} \propto\left(B^{\prime}\right)^{-p}$ with $p=2.2(1)$. For a type-I Rb MOT, operated with $B^{\prime} \approx 10 \mathrm{G} / \mathrm{cm}$, the density limit is about $2 \times 10^{10} \mathrm{~cm}^{-3}$. Using a compressed MOT (CMOT), this can be increased to $5 \times 10^{11} \mathrm{~cm}^{-3}$ by ramping up $B^{\prime}$ to values similar to those we use here [26]. Remarkably, despite its small spring constant (see below), the blue-detuned MOT has $n_{\max }$ similar to that of a type-I CMOT. We attribute this to reduced photon rescattering due to the lower scattering rate.

We turn next to the temperature. Figure 3 shows typical images of an expanding cloud of $N=2.4(1) \times 10^{8}$ atoms released from the blue-detuned MOT. The axial and radial density distributions of these clouds fit well to a Gaussian model. The initial rms widths in these directions are $\sigma_{z}=$ $0.43(2)$ and $\sigma_{\rho}=0.49(2) \mathrm{mm}$, giving a peak density of $n_{0}=N /\left[(2 \pi)^{3 / 2} \sigma_{\rho}^{2} \sigma_{z}\right]=1.5 \times 10^{11} \mathrm{~cm}^{-3}$. The geometric mean temperature found from the expansion is $T=$ 30(1) $\mu \mathrm{K}$. This is lower than typically obtained in a normal $\mathrm{Rb}$ MOT, especially for such a high density. In densitylimited red-detuned MOTs, atoms are heated by photon rescattering [27] and are pushed out by internal radiation pressure to regions of larger magnetic field, where subDoppler cooling is inhibited. In the blue-detuned MOT, the reduced scattering rate reduces this heating and subDoppler cooling is more robust to magnetic fields [13], so lower temperatures can be reached.

Figure 4 shows how the temperature depends on the key parameters. Figure 4(a) shows that the temperature is low for both positive and negative $\delta f_{22}$, but that the lowest
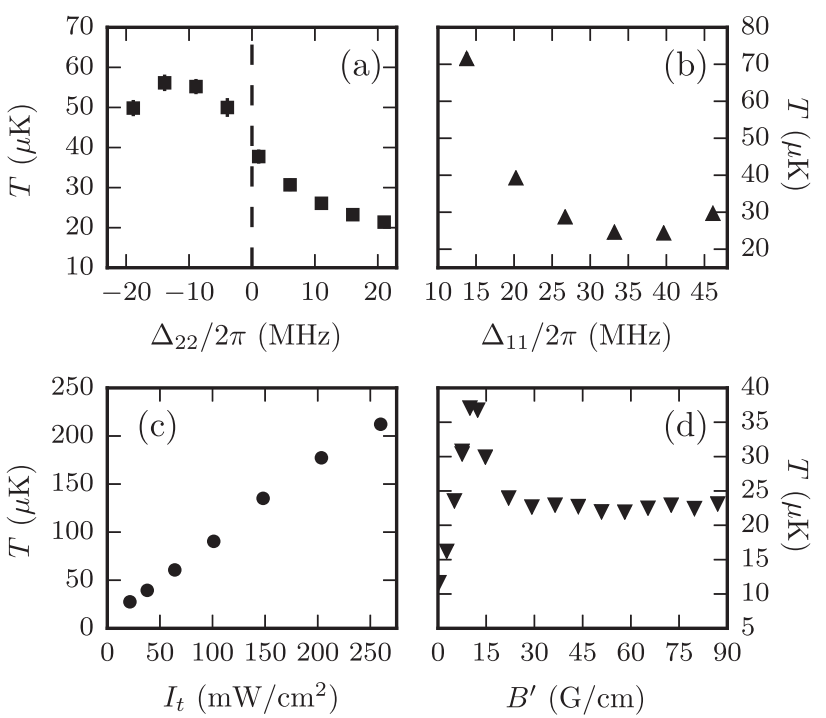

FIG. 4. (a) Temperature versus $\delta f_{22}$ when $\delta f_{11}=35 \mathrm{MHz}$, $I_{t}=21 \mathrm{~mW} / \mathrm{cm}^{2}$, and $B^{\prime}=40 \mathrm{G} / \mathrm{cm}$. (b) Temperature versus $\delta f_{11}$ when $\delta f_{22}=11.5 \mathrm{MHz}, \quad I_{t}=19 \mathrm{~mW} / \mathrm{cm}^{2}$, and $B^{\prime}=$ $40 \mathrm{G} / \mathrm{cm}$. (c) Temperature versus $I_{t}$ when $\delta f_{11}=35 \mathrm{MHz}$, $\delta f_{22}=11.5 \mathrm{MHz}$, and $B^{\prime}=88 \mathrm{G} / \mathrm{cm}$. After loading the MOT at full intensity, $I_{t}$ is held at the reduced value for $5 \mathrm{~ms}$ before measuring the temperature. (d) Temperature versus $B^{\prime}$ when $\delta f_{11}=35 \mathrm{MHz}, \delta f_{22}=11.5 \mathrm{MHz}$, and $I_{t}=28 \mathrm{~mW} / \mathrm{cm}^{2}$.

temperatures are found when $\delta f_{22}>0$, as expected. Figure 4(b) shows that the temperature decreases sharply as $\delta f_{11}$ increases beyond $10 \mathrm{MHz}$, reaching a minimum near $35 \mathrm{MHz}$. We expect the temperature to be inversely proportional to the damping coefficient and proportional to the momentum diffusion constant, which is in turn proportional to the scattering rate. The damping coefficient is predicted to be intensity independent [13], while the scattering rate increases with intensity, so we expect $T$ to increase with $I_{t}$. Figure 4(c) shows that the relation is linear over the range of $I_{t}$ explored. Figure 4(d) shows that the temperature initially increases with $B^{\prime}$, but then gradually declines beyond $15 \mathrm{G} / \mathrm{cm}$, settling to a constant for $B^{\prime}>30 \mathrm{G} / \mathrm{cm}$. This behavior reflects the velocitydependent force curves for various magnetic fields given in Ref. [13]. At low $B$, sub-Doppler cooling relies on motion-induced nonadiabatic transitions between dark and bright states [20], while at higher $B$, Larmor precession between bright and dark states can take over [22,23].

The position- and velocity-dependent forces are quantified in terms of the spring constant $\kappa$ and damping coefficient $\alpha: m \ddot{z}=-\kappa z-\alpha \dot{z}$. We obtain $\kappa$ from the equipartition theorem, which gives $\kappa_{\rho(z)} \sigma_{\rho(z)}^{2}=k_{B} T$. This expression is valid for a harmonic trap in the limit of low $N$, where multiple photon scattering can be neglected. We use the values of $V_{0}$ from the fits shown in Fig. 2 to determine $\sigma_{\rho}^{2} \sigma_{z}$ and use the relation $\sigma_{\rho}=\sqrt{2} \sigma_{z}$ to obtain $\sigma_{\rho}$. Provided the MOT beams are carefully aligned and their intensities 
well balanced, we find this relation to be accurate for low $N$. In this same limit, we measure equal axial and radial temperatures and find that a tenfold increase in $N$ changes $T$ by less than $10 \%$. From measurements of $V_{0}$ and $T$, we obtain $\kappa_{z}=5(2) \times 10^{-20} \mathrm{~N} / \mathrm{m}$ when $I_{t}=113 \mathrm{~mW} / \mathrm{cm}^{2}$, $B^{\prime}=87 \mathrm{G} / \mathrm{cm}, \delta f_{11}=35 \mathrm{MHz}$, and $\delta f_{22}=11.5 \mathrm{MHz}$. The large uncertainty reflects the range of $\kappa_{z}$ measured for various beam alignments and intensity imbalances, all giving small, roughly spherical MOTs. From a theoretical curve similar to Fig. 1(b), calculated for the exact parameters used here, we predict $\kappa_{z}=15(6) \times 10^{-20} \mathrm{~N} / \mathrm{m}$, roughly 3 times larger than measured. The measured value is 50 times smaller than that found for a normal $\mathrm{Rb}$ MOT operated at $B^{\prime} \approx 20 \mathrm{G} / \mathrm{cm}$ [28]. From data reported for a red-detuned type-II MOT of ${ }^{85} \mathrm{Rb}$ [9], we infer $\kappa_{z}=$ $8 \times 10^{-20} \mathrm{~N} / \mathrm{m}$, consistent with our value. Our value is also similar to those found for dc and rf MOTs of SrF and $\mathrm{CaF}$ molecules $[4,6,29,30]$. With $\kappa_{z}$ fixed, we obtain $\alpha$ from the overdamped relaxation of the MOT following a rapid displacement of the trap center. For the same parameters as above, we find $\alpha / m=4.2(6) \times 10^{3} \mathrm{~s}^{-1}$. For these parameters, the theoretical curve similar to Fig. 1(c) gives $\alpha / m=3.9(2) \times 10^{3} \mathrm{~s}^{-1}$, consistent with the measurement.

Figure 5(a) shows how the number of atoms decays over time. We describe the atom loss by

$$
\frac{d N}{d t}=-\gamma N-\beta \int n^{2} d^{3} r
$$

The first term on the right describes loss due to background gas collisions, while the second describes loss due to collisions between trapped atoms. Using a Gaussian density distribution with peak density $n_{0}=N /\left(V_{0}+N / n_{\max }\right)$, we obtain

$$
\frac{d N}{d t}=-\gamma N-\frac{\beta}{2^{3 / 2}} \frac{N^{2}}{V_{0}+N / n_{\max }} .
$$

We fit the data to the solution of this equation, with $V_{0}$ and $n_{\max }$ fixed to the values found from the fits in Fig. 2. The
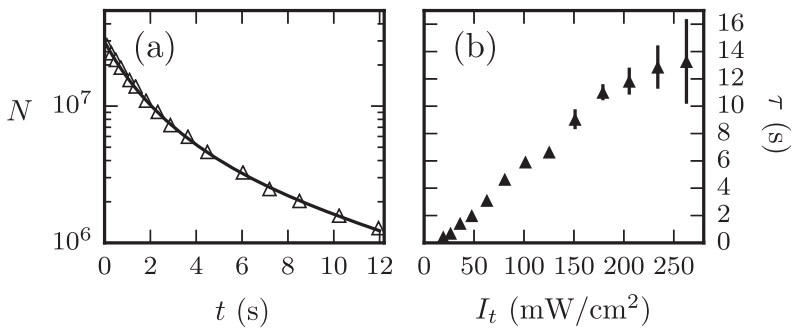

FIG. 5. (a) Number of atoms in the MOT as a function of time. The blue-detuned light is turned on at $t=0$. Parameters are $I_{t}=240 \mathrm{~mW} / \mathrm{cm}^{2}, \quad \delta f_{11}=26 \mathrm{MHz}, \quad \delta f_{22}=12 \mathrm{MHz}, \quad B^{\prime}=$ $48 \mathrm{G} / \mathrm{cm}$. (Line) Fit to the model described in the text. (b) Lifetime $\tau$, measured for small $N$, as a function of $I_{t}$. Parameters are $\delta f_{11}=26 \mathrm{MHz}, \delta f_{22}=12 \mathrm{MHz}, B^{\prime}=39 \mathrm{G} / \mathrm{cm}$. data fit well to this model, giving $\gamma=0.075(3) \mathrm{s}^{-1}$ and $\beta=1.75(3)(35) \times 10^{-10} \mathrm{~cm}^{3} \mathrm{~s}^{-1}$. For $\beta$, the first bracketed number is the statistical uncertainty in the measurement, while the second is the systematic uncertainty due to the uncertainty in $N$. In the limit of small $N$, the trap lifetime is $\tau=1 / \gamma=13.3(5) \mathrm{s}$. This is about 4 times longer than the characteristic loading time of the type-I MOT for the same $I_{t}$, implying that the lifetime of the blue-detuned MOT is longer than the type-I MOT at this $I_{t}$. This is consistent with our observation that the excited-state fraction in the bluedetuned MOT is about a quarter of that of the normal MOT, together with the previous observation that collisions with background $\mathrm{Rb}$ eject excited-state atoms at 3 times the rate of ground-state atoms [31]. Figure 5(b) shows how the lifetime depends on $I_{t}$ in the low- $N$ limit. The observed increase of $\tau$ with $I_{t}$ is surprising, since the trap depth is far smaller than the temperature of the background gas, but may be due to glancing collisions that are effective at ejecting atoms at low $I_{t}$, where the trap depth is low, but less so as $I_{t}$ increases. A similar observation has been noted for a normal MOT at low intensity [32]. The coefficient $\beta$ has been studied previously in ${ }^{87} \mathrm{Rb}$ MOTs [32,33]. Values similar to ours were measured at low intensity, where the trap depth was lower than the energy released in a hyperfine-changing collision. With increasing intensity, $\beta$ was observed to fall as the trap depth increased, but then increased again as collisions between ground and excited atoms started to dominate. For intensities exceeding $15 \mathrm{~mW} / \mathrm{cm}^{2}$, a roughly constant $\beta \approx 2 \times 10^{-12} \mathrm{~cm}^{3} \mathrm{~s}^{-1}$ was measured, 100 times smaller than our value. We suggest that hyperfine-changing collisions dominate in our MOT for all $I_{t}$, because of its low trap depth, and that this is the reason for the large $\beta$. For a red-detuned type-II MOT of ${ }^{85} \mathrm{Rb}, \beta=9.1(7) \times$ $10^{-9} \mathrm{~cm}^{3} \mathrm{~s}^{-1}$ has been reported [9].

The MOT shown in Fig. 3 has a phase-space density of $6 \times 10^{-6}$, the maximum we have observed in these experiments. This is a million times higher than previously reported for a red-detuned type-II MOT of Rb [9]. For molecular MOTs, which always use type-II transitions, a switch from red to blue detuning may offer a similar enhancement in phase-space density. The blue-detuned MOT also compares favorably to type-I alkali MOTs, where the highest phase-space densities are obtained using the dark-SPOT technique [14]. Our phase-space density is about the same as the highest achieved by that method [15]. Even higher phase-space density was recently achieved for Sr atoms by using several stages of laser cooling [34]. Bluedetuned MOTs seem advantageous for efficient loading of atoms and molecules into conservative traps, including optical tweezer traps and chip-scale traps, where dissipation, low temperature, and tight confinement are all needed. Our MOT offers new opportunities to study the control of ultracold collisions, especially the suppression of inelastic processes by optical shielding [35-37], which should be effective for near-resonant blue-detuned light. It can also 
serve as a good starting point for evaporation of atoms and molecules to quantum degeneracy.

Data underlying this article can be accessed from Zenodo at Ref. [38], and may be used under the Creative Commons CCZero license.

We are grateful for helpful discussions with Ed Hinds and Jeremy Hutson. This research has received funding from EPSRC under Grants No. EP/M027716/1 and No. EP/ $\mathrm{P} 01058 \mathrm{X} / 1$.

*k.jarvis14@imperial.ac.uk

[1] E. L. Raab, M. Prentiss, A. Cable, S. Chu, and D. E. Pritchard, Phys. Rev. Lett. 59, 2631 (1987).

[2] L. D. Carr, D. DeMille, R. V. Krems, and J. Ye, New J. Phys. 11, 055049 (2009).

[3] J. F. Barry, D. J. McCarron, E. B. Norrgard, M. H. Steinecker, and D. DeMille, Nature (London) 512, 286 (2014).

[4] E. B. Norrgard, D. J. McCarron, M. H. Steinecker, M. R. Tarbutt, and D. DeMille, Phys. Rev. Lett. 116, 063004 (2016).

[5] S. Truppe, H. J. Williams, M. Hambach, L. Caldwell, N. J. Fitch, E. A. Hinds, B. E. Sauer, and M. R. Tarbutt, Nat. Phys. 13, 1173 (2017).

[6] L. Anderegg, B. L. Augenbraun, E. Chae, B. Hemmerling, N. R. Hutzler, A. Ravi, A. Collopy, J. Ye, W. Ketterle, and J. M. Doyle, Phys. Rev. Lett. 119, 103201 (2017).

[7] M. Prentiss, A. Cable, J. E. Bjorkholm, S. Chu, E. L. Raab, and D. E. Pritchard, Opt. Lett. 13, 452 (1988).

[8] J. Flemming, A. M. Tuboy, D. M. B. P. Milori, L. G. Marcassa, S. C. Zilio, and V. S. Bagnato, Opt. Commun. 135, 269 (1997).

[9] V. B. Tiwari, S. Singh, H. S. Rawat, and S. C. Mehendale, Phys. Rev. A 78, 063421 (2008).

[10] B. K. Stuhl, B. C. Sawyer, D. Wang, and J. Ye, Phys. Rev. Lett. 101, 243002 (2008).

[11] M. R. Tarbutt, New J. Phys. 17, 015007 (2015).

[12] M. R. Tarbutt and T. C. Steimle, Phys. Rev. A 92, 053401 (2015).

[13] J. A. Devlin and M. R. Tarbutt, New J. Phys. 18, 123017 (2016).

[14] W. Ketterle, K. B. Davis, M. A. Joffe, A. Martin, and D. E. Pritchard, Phys. Rev. Lett. 70, 2253 (1993).

[15] N. Radwell, G. Walker, and S. Franke-Arnold, Phys. Rev. A 88, 043409 (2013).

[16] C. Valentin, M.-C. Gagné, J. Yu, and P. Pillet, Europhys. Lett. 17, 133 (1992).
[17] D. Boiron, C. Triché, D. R. Meacher, P. Verkerk, and G. Grynberg, Phys. Rev. A 52, R3425 (1995).

[18] A. Hemmerich, M. Weidemüller, T. Esslinger, C. Zimmermann, and T. Hänsch, Phys. Rev. Lett. 75, 37 (1995).

[19] A. T. Grier, I. Ferrier-Barbut, B. S. Rem, M. Delehaye, L. Khaykovich, F. Chevy, and C. Salomon, Phys. Rev. A 87, 063411 (2013).

[20] M. Weidemüller, T. Esslinger, M. A. Ol'shanii, A. Hemmerich, and T. W. Hänsch, Europhys. Lett. 27, 109 (1994).

[21] S.-Q. Shang, B. Sheehy, P. van der Straten, and H. Metcalf, Phys. Rev. Lett. 65, 317 (1990).

[22] O. Emile, R. Kaiser, C. Gerz, H. Wallis, A. Aspect, and C. Cohen-Tannoudji, J. Phys. II (France) 3, 1709 (1993).

[23] R. Gupta, S. Padua, C. Xie, H. Batelaan, and H. Metcalf, J. Opt. Soc. Am. B 11, 537 (1994).

[24] T. Walker, D. Sesko, and C. Wieman, Phys. Rev. Lett. 64, 408 (1990).

[25] D. W. Sesko, T. G. Walker, and C. E. Wieman, J. Opt. Soc. Am. B 8, 946 (1991).

[26] W. Petrich, M. H. Anderson, J. R. Ensher, and E. A. Cornell, J. Opt. Soc. Am. B 11, 1332 (1994).

[27] G. Hillenbrand, C. J. Foot, and K. Burnett, Phys. Rev. A 50, 1479 (1994).

[28] C. D. Wallace, T. P. Dinneen, K.-Y. N. Tan, A. Kumarakrishnan, P. L. Gould, and J. Javanainen, J. Opt. Soc. Am. B 11, 703 (1994).

[29] D. J. McCarron, E. B. Norrgard, M. H. Steinecker, and D. DeMille, New J. Phys. 17, 035014 (2015).

[30] H. J. Williams, S. Truppe, M. Hambach, L. Caldwell, N. J. Fitch, E. A. Hinds, B. E. Sauer, and M. R. Tarbutt, New J. Phys. 19, 113035 (2017).

[31] M. H. Anderson, W. Petrich, J. R. Ensher, and E. A. Cornell, Phys. Rev. A 50, R3597 (1994).

[32] C. D. Wallace, T. P. Dinneen, K.-Y. N. Tan, T. T. Grove, and P. L. Gould, Phys. Rev. Lett. 69, 897 (1992).

[33] S. D. Gensemer, V. Sanchez-Villicana, K. Y. N. Tan, T. T. Grove, and P. L. Gould, Phys. Rev. A 56, 4055 (1997).

[34] S. Bennetts, C.-C. Chen, B. Pasquiou, and F. Schreck, Phys. Rev. Lett. 119, 223202 (2017).

[35] S. Bali, D. Hoffmann, and T. Walker, Europhys. Lett. 27, 273 (1994).

[36] V. Sanchez-Villicana, S. D. Gensemer, K. Y. N. Tan, A. Kumarakrishnan, T. P. Dinneen, W. Süptitz, and P. L. Gould, Phys. Rev. Lett. 74, 4619 (1995).

[37] K.-A. Suominen, J. Phys. B 29, 5981 (1996).

[38] https://doi.org/10.5281/zenodo.1134648 